\title{
AIP and the Intersubjective: Implications for Practice and Training in EMDR - Part One
}

\section{Abstract}

Developmental and interpersonal neurobiology adds the inter subjective dimension to AIP and memory networks both adaptive and dysfunctional. This dimension begins through early interactional patterns between caretaker and infant, and has been linked to the patterns of interaction between client and clinician. The mirror neuron system, elaborates the inter subjectivity of these networks. AIP, guides EMDR practice, and sets the groundwork with a memory network/information processing framework which allows an elaboration of this inter subjective matrix. This elaborated framework suggests the need to expand instructions to clinicians to include their activated memory networks as intrinsic to the inter subjective matrix in EMDR. An explication of these issues within this framework can allow clinicians a greater understanding of their role in the therapeutic process, and suggests ways clinicians can best self monitor and make needed adjustments to expedite their clients' progress.

Keywords: Adaptive information processing; Inter subjective; Mirror neurons
Review Article

Volume 10 Issue 3 - 2017

Mark Dworkin*

EMDR Trainer, USA

*Corresponding author: Mark Dworkin, LCSW, PC, $10^{\text {th }}$ Awardee Francine Shapiro Lifetime Achievement Award, EMDR Trainer, USA, Email: markdworkinlcsw@gmail.com

Received: December 07, 2016 | Published: December 29, 2017

\section{Introduction}

Primary inter subjectivity [1] occurs at birth as babies enter the world helplessly, except to cry and be cared for. Adaptive and dysfunctional memory networks then develop through functional and dysfunctional transactions between caretaker and infant experience. During the first eighteen months of life, experiences are encoded implicitly forming the foundation of these networks. "Human connections create neuronal connections [2]." Corticolimbic connections during this period of postnatal development have been demonstrated in the development of affect regulation memory networks as a result of positive attachment experiences [3-5]. Developmental overpruning and death of neurons responsible for these connections hamper the development of affect regulation [6]. Without positive attachment experience the amygadala, termed the "fear center" will become hyperactivated in the face of perceived threat [7]. Researchers have discovered that mirror neurons activate motor neurons when attuned to an intention of another. Action observation induces action intention, experienced physiologically as an as an embodied simulation of the "other" in the infant/child/adult brain. The observation/intention process becomes encoded in the memory networks of the brain and may become activated during moments that are associated with prior implicitly encoded memories [8-19].

The research on the mirror neuron system, resonance circuit of the social brain has implications for the treatment of traumatized clients with EMDR. Starting wit5h the beginning of life, through repetitive cycles the infant begins to learn within an inter subjective matrix, "our nervous systems are constructed to be captured by the nervous systems of others" with the discoveries of the mirror neuron system as its neurobiological base. The intersubjective matrix is encoded in memory networks through all significant life experiences. It is through this process that empathy, defined as a matching of inner and outer and outer experience, is developed. Through empathy we "know" the experience of the other through our internal experiences of them $[14,16,17,20,21]$.

These complex inter subjective matricies are implicitly described in AIP. Adaptive or dysfunctional patterns of secure or insecure attachment resonances continue through the life span [2,3,22-31]. These neurobiological processes within an AIP framework facilitates clinician's understanding of when to stay out of the way, or intervene to facilitate blocked reprocessing. Developmental neurobiology, and mirror neuron research demonstrates that the clinician's memory networks are always interacting with the client's; hence EMDR protocols and procedures cannot be set apart from the memory networks of client and clinician.

The term "inter subjective matrix" defined by Stern will be used to elaborate interactions of adaptive and dysfunctional memory networks between clinician and client. The reader, familiar with former works of the author, will notice that the term "therapeutic relationship" is conspicuously absent. This term appears to confuse more than it clarifies. It seems to indicate a warm and understanding relationship that may be a vehicle for imparting coping strategies [32], or for respecting the inherent humanity of the traumatized client, without much use for resolving the effects of trauma (personal communication Bessel van der Kolk 2008). Instead, Dr. van der Kolk and I agreed last summer on the utility of the term "resonance" (or in Siegel's terms, mental state resonance (1999). Concepts such as the collaborative working alliance [33]; rupture and repair [34]; implicit relational knowing [35], now moments and moments of meeting, are terms used to illustrate what occurs inter subjectively in EMDR psychotherapy. Finally, as the author is male, references to client and clinician are in the 
same gender. No disrespect is intended. It is simply a matter of the author's need to stay focused. Everything EMDR starts with the Adaptive Information Processing model [36-41].

\section{The Adaptive Information Processing Model}

The Adaptive Information Processing (AIP) Model posits that, "there exists a distinct balance in neurological systems that allow information to be processed to an adaptive conclusion," and that pathology is, "the result of unprocessed experiences, stored in their own neural networks, unable to link up naturally with anything more adaptive." [36-41]. Since EMDR psychotherapy is guided by AIP it follows that if unprocessed memory is pathology, it would seem that applying the procedures of EMDR with fidelity to the methodology will yield the most robust results [42]. Their conclusions are based on research studies, not on the complex processes of the daily interactions clinicians face with clients who do not meet research criteria. Their Meta analyses include studies that have not included this inter subjective matrix. While the AIP model has been explained fully in previous works this paper adds to the professional literature by elaborating the complexity of the inter subjective matrix in the daily practice of EMDR psychotherapy. These matricies affect all interactions between client and clinician and include all present moment processes when protocols and procedures, such as developing Targeting Sequence Plans, strategies; the Safe/Calm Place procedure; the procedural steps outline; facilitating blocked processing; "staying out of the way"; cognitive interweaves; instructions installing positive cognitions, a body scan, closing down an incomplete session, and reevaluation (2001).

\section{Clinical Judgment in EMDR}

Shapiro stresses that EMDR is not a "cookie cutter" approach and must be tailored to each client" (Shapiro 2001 p 381); clinical judgment needs to be applied during all phases (Shapiro 2001), and that clinician factors affect EMDR methodology. Now through the research of neuroscience clinical judgment in EMDR now can be expanded to include understanding attachment processes between client and clinician, and identifying and intervening in this inter subjective matrix. The elaboration of instructions to the clinician now includes the non linear memory network activations and the role of the clinician's memory networks in this process. The use of the term "counter transference (EMDRIA 2007) is problematic. It is defined as interference, as first used by Freud in his classical definition of the term (Freud 1912). Other theorists began to wonder if counter transference can be used productively in treatment of the patient [43-48] as in the totalistic, and integrative views. This author has defined countertransference in information processing terms as, "the activation of state dependent memories in the clinician that have been sparked by the client, intentionally or not [49]." As every memory network activation. Memory network activations are the root of attunement and resonance, necessary for the practice of EMDR. The term should not imply pathological processes (as suggested in Shapiro 2007a,b, and the guideline of EMDRIA's criterion for teaching EMDR), because implicitly activated clinician memory networks are sources of information of non conscious messages clients are transmitting affectively. Identifying and decoding them can enhance positive outcomes through relational interweaves [48].
Activated clinician memory networks are part of a neurobiological inter subjective matrix at all moments of EMDR psychotherapy.

\section{Attachment and the Inter subjective Matrix: Issues between Client and Clinician}

Attachment research has been informative in explaining the nature of clinician/ client relatedness. "Attachment is based upon collaborative communication" [2], and in secure attachment patterns, contingent communication between caretaker and infant helps shape the developing brain. Secure attachment experiences form the basis of adaptive memory networks. The theory of interpersonal neurobiology rests on definition of memory, congruent with AIP, in which past events influence future function." The HPA axis activities of securely attached children do not show elevations in cortisol levels, showing that they have begun to develop fear arousal strategies. Their corticolimbic neural circuitry has developed sufficiently by eighteen months to provide a buffer against distress of separation without the elevation of glucocorticoids (Spangler and Grossman 1990). In contrast, those infants with depressed or disorganized caretakers develop insecure attachment patterns, dysfunctional memory networks, and have elevated cortisol levels responsible for the developmental over pruning Perry refers to. The stress response system of insecure attachment patterns reveals an underactive or overactive pattern of dysfunctional memory networks inadequate for reducing fear responses. These early attachment patterns may be repeated in the client clinician encounter, needing the formation or strengthening of resources and positive experience before active reprocessing strategies of traumatic experiences to safely begin $[50,51]$. The connection between attachment and intersubjectivity highlights the role of reciprocal mutual influence, and vulnerabilities of the client and clinician that are embedded in this matrix [2].

The clinician's attachment patterns, inter subjective consciousness, memory networks, and information processing system differ from those of his client's. Each makes ongoing appraisals of the other's intentions, and when there may be perceived or actual misattunements, ruptures to the therapeutic bond may occur and significant complexities may arise Strengthened therapeutic attachment patterns, or therapeutically "implied relational knowing" [35] need to be firmly established for a good enough alliance that will carry through, and be tested, during active trauma reprocessing phases [39,48,51-54]. Instructions for maintaining optimal inter subjective interactions in EMDR need to be supplemented with the continual examination and self reflection of one's own attachment processes visa vie the client, the client's attachment processes visa vie the clinician [48]. The attachment literature, starting with Bowlby's three volume work, Attachment and Loss [22-25,35,55-59], all demonstrate that attachment patterns that begin in infancy carry over to the therapeutic alliance.

Consider the similarities of these research conclusions and clinical observations with AIP. "Pathology is the result of unprocessed memories [41]. The infant develops dysfunctional memory networks based upon the interactive nature of the "affectively charged" communications with the caregiver. Mirror neuron research shows that humans respond with intentional 
attunement and embodied simulation to affectively charged actions of the significant other; there is a crossover of between attachment, developmental neurobiology, inter subjectivity and mirror neuron research, and AIP.

The addition of interactions of memory networks with the protocols and procedures of EMDR whether adaptive or dysfunctional; elaborates the basic tenants of AIP. Adaptive to adaptive connection activates the resonance circuit of the social brain $[16,17]$, causing mental state resonance [2] with collaborative contingent communication. When this coconstructed state is stable, the protocols and procedures of EMDR Reprocessing can be applied to the client with little problem, and productive reprocessing of trauma can continue to an adaptive conclusion.

Moving Along, Implicit Relational Knowing, Now Moments and Moments of Meeting: Inter subjectivity Meets AIP

The concept of "moving along" [60] can be captured in the initial stages of EMDR, of the client feeling well received by an attuned and resonant clinician, ready to be of assistance. However, the influences of client's attachment memory networks on the clinician's attachment memory networks may be misattuned when the "implicit relational knowing" of each intersect in a way that activates dysfunctional memory networks of insecure attachment patterns in one or both of them $[26,27,35]$. The term, implied relational knowing" implies a non conscious, procedural way of knowing how to be with one another, based upon repeated interactions with important figures in one's life. A traumatized person may have the implicit relational knowing that "people think I am stupid; they get frustrated with me, and I can't trust them;" this belief may cause them to behave in many dysfunctional ways. One pattern may be over compliance or subservience ("I am trying to please you so you will not be angry with me, but I am too stupid to know how"). An EMDR clinician may have the implicit relational knowing ("People treat me like I don't matter and don't listen to me"). They may have beliefs that cause them to behave in frustrated and irritable manners ("He is treating me this way because I am unimportant.")

This clinician's initial responses to the client who acts subserviently may begin by acting "kindly" (since the client obviously is trying without success) possibly until their frustration starts to become reflected in the mirror neurons of the client who may then withdraw into a dysfunctional belief of "I can't do this", or, "I'm incompetent;" the clinician's mirror neuron activation may cause him to have an activated memory network of being injured "how can you be so stupid?)" Or (I'm not worth listening to").

Client and clinician are neurobiologically connected in a two person, subject to subject, in an ongoing bidirectional, nonlinear experience. An example during the History Taking phase may be found on page 22 of the March 2009 Part One Training Manual, "Developing Appropriate Negative Beliefs (Cognitions NC)."

Gary is a 28 year old male patient who is single and working as a teacher for special needs children. He recently received a poor evaluation by a supervisor, and became quite anxious believing that he would lose his job for being stupid. The EMDR clinician evaluates the client's whole clinical picture and begins to develop a Targeting Sequence Plan. Starting with the present day trigger the client says that the image he sees is of his supervisor frowning. The current instructions next call for obtaining a negative cognition. They read, "When you think about of (repeat description... supervisor's frown...) what negative belief do you have about yourself? The clinician may receive this reply, "I feel ashamed of myself." The clinician then responds, "What does that say about you as a person?" The client responds, "I hate myself;" Now the clinician starts to become a little frustrated and says (according to the manual) "What negative belief goes with that emotion ("of hating yourself"?) Well, I have hated myself since I've been a child." The frustration (and failure memory networks of the clinician now become more pronounced) and the clinician replies back to the client (with a little irritation starting to creep into his voice), "not what you believed about yourself then, what do you believe about yourself now?" The clinician's frustration continues to increase, and he says, with irritation in his voice, "Gary, when you see your supervisor's frown, in your worst moment, what negative belief you have about yourself when you think of that event?" Gary's auditory mirror neurons sense the clinician's irritability and his failure memory networks become activated by the clinician's frustration and he responds, "I'm doing this all wrong now aren't I?"

This previous dialogue is an example of a rupture of attunement. The clinician's visual and auditory neurons now become activated empathically to his client's activated failure memory networks and his adaptive memory networks containing compassion become activated and he responds, "I know that this question is difficult for you to answer the way I want you to; is it possible that my responses to you made you feel like you were failing again?" Gary shrugs his shoulders and nods. "So maybe the questions, or the way I was asking them bothers you now, so it's possible that you experiencing your negative beliefs now. Let's see if we could try this again a little differently. Would that be OK?" Both experience relief in this moment, and they repair this rupture. Implicitly, the clinician is letting the client (and himself) know that it is $\mathrm{OK}$ to make mistakes (hence mistakes do not equal failure and I am here for you.). This is a "moment of meeting" that repairs the rupture of attunement. Is this transference and counter transference? Maybe, but it might be better explained as the interaction of activated adaptive and dysfunctional memory networks, with mirror activations occurring in each party, and self corrections happening as each party looks at what their part in the process might have been. The standard EMDR instruction to, "strive for optimal attunement," may be better modified to suggest that the clinician mindfully notice how his subjectivity (memory networks) starts and continues to be of influence to the client's subjectivity (memory networks.)

The preceding example was of a "now moment" and "moment of meeting." A now moment is "an affectivity charged moment because it puts the nature of the client clinician relatedness into question," (Stern 2004). Overtly, the clinician is following the standard instruction eliciting a negative belief during the Targeting Sequence Plan. However, while Gary is trying to please the clinician by following the instruction, in parallel process it exposes his implied relational knowing to the clinician, "in the moment," that "people think I'm stupid (and therefore I'm 
ashamed of myself)..." "Moments of meeting" involves a response that is well fitted to the particular crisis. It cannot be a general technical response..." The instructions, "When you think about yourself, ...what negative belief do you have about yourself as a person;... what does that say about you as a person; what negative belief goes with that emotion...?" etc, are all general technical responses, and therefore do not meet criterion for a moment of meeting.

This now moment, puts Gary's and the clinician's alliance in jeopardy, and an "a well fitted response" (moment of meeting) is needed in order to solve the inter subjective crisis. At first they both enacted the exact problems Gary and the clinician both experienced, only this time it happened "in the moment." The EMDR clinician needs to become aware of this repetition, with awareness of his dysfunctional memory network activations; otherwise Gary's implied relational knowing ("people think I am stupid; they get frustrated with me, and I can't trust them ;) will be reinforced. Another failed dyadic experience will occur because the clinician's implied relational knowing ("people treat me like I don't matter and don't listen to me"). And his negative cognition "I am unimportant" is actually a defense against a similar implied relational knowing of Gary's, on the clinician's part.

The clinician's response does not overtly meet the required instruction, ("what negative belief do you have about yourself") but is "well fitted to the situation is the "moment of meeting" which engages Gary in a co-created problem solving attempt. This moment of meeting might involve the clinician's awareness of the dyadic process through his somatic reactions. A somatic reaction (physiologic activation or numbing of a dysfunctional memory network) is the best clue a clinician can use to begin the process of self correction. It may be that the working out this dilemma could be found in the reenactment between client and clinician $[61,62]$. The client's original (unspoken, and unthought) implicit dysfunctional memory network activation may also include, "I don't want to believe anything bad about myself now. It will hurt too much." This part of the memory activation never becomes part of the client's explicit verbalizations; he just feels badly, reacts in the way that is consonant with his implicit relational knowing, and now moments continue to develop without "negotiated repairs to the rupture of the therapeutic alliance. Eventually the odds that EMDR psychotherapy will be a successful joint venture will continue to diminish. The client and clinician need to identify these now moments; notice what the experience like for each when the work becomes blocked; and find an adaptive link to this dysfunctional moment. While this is an example of what may occur in the History Taking phase, these potential inter subjective problems may occur in every phase of EMDR.

\section{Now Moments and Moment of Meeting During the Desensitization Phase}

These "now moments" can be observed during the desensitization phase of EMDR clinical practice when the client reports that "nothing" has occurred after two consecutive sets of EM's. While there are many "instructive" procedures to facilitate blocked processing; careful attention needs to be paid, by the clinician to the verbal or non verbal inter subjective experiences that may be occurring when reprocessing trauma becomes blocked. The adaptive and dysfunctional memory networks of both client and clinician; both with mirror neuron activations, and somatic reactions (whether somatic activations, or somatic numbing) can guide the clinician in making these clinical judgments.

Robert, a 36 year old, single, social worker, contacted me for EMDR reprocessing. He had been traumatized by a harsh romantic break up and believed that he was a failure because of this rejection. Other treatment methods were not useful in helping him resolve his dysfunctional failure and rejection memory networks, and he decided that he would "try" EMDR. His implicit relational knowing was that older men show kindness and compassion. He reported a good relationship with his father; a fair relationship with his mother ("she's a bit too emotional for my taste, just like most women"), and he had a strong social support network. We made a good beginning alliance; he informed me during history taking that there were few childhood "issues"; scored low on his DES-T, and completed all procedures of the Preparation phase. While I had my suspicions that there were more childhood issues than he was aware of, it seemed as though we had mental state resonance, and he had the requisite coping abilities to begin the active phases of EMDR.

He reprocessed some childhood issues of rejection during athletic competitions with little distress. During one of these reprocessing sessions he began to remember a dissociated memory of being a little child and finding out that his mother, who was not present to watch him play little league, had an extramarital affair with his father's best friend. We decided to focus on this trauma next after finishing his childhood athletic competition trauma.
P: "I see my father crying and I go over to comfort him.
NC: "I am a failure"
PC: "I did the best I could"

VoC: 3

E: Sadness and guilt

SUDS: 8

\section{Body: Heartache and pain in his stomach}

At the end of two sets of EM's he informed me that no new information had come up for him. I used longer sets, change of direction, instructions for under accessing a target memory, and cognitive interweaves such as "If this had happened to your best friend as a little boy, what would you tell him? No "standard instruction" seemed to fit this now moment. At that moment I had an experience of not being able to think clearly. This is an example of an inter subjective "now moment." I quickly reflected on this dilemma; and wondered to myself if my mirror neuron system was sensing non conscious dysfunctional memory networks from Robert which may have been activating dysfunctional unprocessed memories of my own when I felt helpless and could not figure out what to do. 
I first compartmentalized the anxiety I felt in not being able to think clearly; then I decided to share my inner experience of clouded thinking as a "moment of meeting." (My implicit relational knowing was that I am safe to reveal my process). When I informed him of my inner experience he exclaimed, "That's it! That's what I experienced. I had forgotten all about it." I couldn't think clearly and I blanked out." I suggested that we reprocess this inter subjective moment of what had just happened between us, and another dissociated memory network of Robert's became activated. He remembered an experience of being six years old, in his first school play and forgetting his lines. His mother, who was in the audience, began to cry, activating shame in Robert, and causing him to urinate in his pants, increasing his feelings of humiliation to unbearable levels. He experienced an elongated period of intense emotional releases with dual attention, and verbal support from me. After this period of release he recognized that he had been choosing women who would be critical of him to date. It took us three 90 minute sessions to reprocess the rest of his past problems with women, the romantic breakup which had sent Robert to me, his present day referents (dating women with similar characteristics to his mother, and his future template of finding a more appropriate partner whom he eventually married.

\section{The Limitations Of Current Instructions To Clinicians}

In every phase of EMDR instructions are given to the clinician regarding how to perform procedures. An example during the preparation phase would be the train metaphor, "In order to help you "just notice" the experience," imagine riding on a train and feelings, thoughts, etc., are just the scenery going by Shapiro F [21]". Standard instructions are given in all phases; and in the three pronged approach as well. Another example when reprocessing is blocked is "scanning. "The clinician should ask the client to scan the original incident for something that is currently more upsetting than the original target [21]." While useful it does not specify that the clinician's memory networks are in intrinsic relationship with the client's. The clinician has adaptive and dysfunctional memory networks which interact with the client's memory networks, and the procedural elements he is applying, accessing, and stimulating the dysfunctionally stored information is more complex than just following these instructions. A fuller instruction might be added to include, "and just notice mindfully how your attunement is affected, both adaptively and dysfunctionally by your memory network activations and interactions as well as your client's".

Instructional behaviors have utility. It is assumed that clinicians who start EMDR training are already familiar with concepts such as the collaborative working alliance [33] with shared goals, differentiated tasks, and initial bonding. Instructions give the clinician a "roadmap" to follow with all the tasks required of him and his client, and of EMDR's protocols and procedures. During intense emotional releases "The client relies on the clinician to provide emotional stability and a sense of safety during the abreaction" [36]. The clinician is encouraged to continue bilateral stimulation until the client reaches their next plateau of adaptive information processing. Some EMDR clinicians may be tempted to stop bilateral stimulation fearing that the intense release of emotion may be too much for the client, when in actuality the dysfunctional memory networks of the clinician may become activated, and this release may be too intense for the clinician. The procedure during the preparation phase of learning and practicing the Stop Signal is designed to give the client power to limit the amount of discomfort they can bear.

Clinicians' whose memory networks become dysfunctionally activated during intense emotional reactions may have beliefs that they are "harming" the client by continuing bilateral stimulation, when the client seems to be adaptively remaining in dual attention, but has not reached the next plateau of adaptive information processing. Inter subjectively activated dysfunctionally unprocessed memories of the clinician may be interpreted (accurately or inaccurately) as doing harm in the present. The implication is it is the clinician that has lost dual awareness. The reader may interpret this clinician activation of his dysfunctional memory networks as "counter transference" that needs to be dealt with so that the clinician does not interfere with the client's reprocessing. The inter subjective matrix is far more complex. However, the clinician may be assisted by an additional instruction of, "and as long as your client continues in dual attention and also show progressions in their information progressing, then continue reprocessing their dysfunctional memory networks; AND mindfully notice your memory activations and use the compartmentalization strategies you developed to help calm those reactions." As instructions currently exist the clinician is told to "keep the car moving all the way through the tunnel [63], perhaps with the minor interventions "it's old stuff," "just notice it." Instructions to, "stay out of the way" when information processing is occurring "adaptively"; facilitate blocked processing to "mimic spontaneous processing;" or by intervening with a cognitive interweave to link in an adaptive response to a dysfunctional memory network [63] are useful examples of instructions to clinicians; they are just limited in their focus.

Current instructional guidance aids critical thinking, but ignores the possibility that part of the blocks in reprocessing may be caused by implicit nonlinear interactions. The clinician is intrinsically part of the process whether he verbalizes anything or not. Both are in nonlinear continuous inter subjective feedback loops. The resonance circuitry of the social brain "has been shown not only to encode intention (memory networks), but also to be fundamentally involved in human empathy, and also in emotional resonance, the outcome of attunement of minds" [17].

The literature on the neurobiological social connectivity between humans shows that much of inter subjective connectivity is non conscious $[4,13,20,21,35,64]$. Instructions to clinicians to be mindful of their somatic reactions as well enlarges the focus of attention on both client and clinician memory network activations. The example of my becoming unable to think straight while reprocessing a memory with Robert illustrates this point. When the resonance circuits of client and clinician are in positive attunement, then the clinician can follow standard procedure, "staying out of the way" or facilitates blocked processing, and the traumatized client will reprocess dysfunctional memory networks. 
The studies of the mirror neuron system, and developmental neurobiology, and attachment literature have shown that when caretaker and child, or clinician and client engage in goal directed behavior, each participant's memory networks are activated (i.e. the caretaker's response to the baby's cry; the clinician's response to the client reporting what comes up during reprocessing.) When memory networks are activated, behaviors vary. Any parent who tells their child that it's time to go to school or do homework knows that both parent and child have multiple activations, parallel and sequential; bidirectional, and non linear. This analogy applies to EMDR psychotherapy. Whether the EMDR clinician verbalizes, or doesn't verbalize; sit in "ships in the night" (so that the clinician's face is out of the client's line of sight), or has the client have their eyes open or closed, these adaptive and dysfuctional inter subjective matricies occurs.

The semiotics of communication, starting with the observations of the philosopher John Locke [65], are such that even the slightest non verbal change in breathing, facial expression, tonal expression, or any other non verbal communication may be noticed by either party, and can have an effect on both parties:

"All that can fall within the compass of human understanding, being either, first, the nature of things, as they are in themselves, their relations, and their manner of operation: or, secondly, that which man himself ought to do, as a rational and voluntary agent, for the attainment of any end, especially happiness: or, thirdly, the ways and means whereby the knowledge of both the one and the other of these is attained and communicated" [65].

Shin et al. [20] noted that it took a research group of patients with PTSD 33 milliseconds to register an angry face in their amygdala region of their limbic system.

Return to the EMDR clinician who becomes fearful that he has harmed his client during desensitization, and stops bilateral stimulation. The clinician's memory activations may have prompted him to stop bilateral stimulation, because he may have had somatic activations of guilt. He realized that his sadistic networks were activated due to resonating with the client's dysfunctional memory network activation of being emotionally abused by his father. The clinician's activated memory networks containing guilt (adaptively) were informing him that he had been colluding with the client's dissociated memory activation that he needed to be punished in order to keep his father's love. In this case the clinician may then use the client's history to realize that he may be repeating the abuser/victim pattern in this "in the moment process" of continuing bilateral stimulation. If this were the case it might prompt the clinician to question the client if what he was experiencing was similar these old abuse memories. Perhaps by beginning this process by stating, "I'm puzzled, it feels like you and I am repeating an old problem, does it feel like that to you?" An additional strategy might be to suggest to the client to scan his body while asking himself the question. If the client were able to make the connection, the clinician could accept rational responsibility of having been part of this repetition without any content revelations) and ask the client to process this inter subjective process; in this case the client may then successfully reprocess this old memory successfully, or if he became blocked again it might be possible to ask the client what he would say to his best friend if he believed that he deserved to be punished in order to keep his father's love. Reprocessing could then continue with reasonable progressions. The first block to reprocessing might have been the reenactment of client and clinician in continuing bilateral stimulation when progressions were not happening.

The second block to reprocessing may not be due to an inter subjective experiencing. In this case the clinician would experience his adaptive memory networks in attunement with his client. Then one of the standard cognitive interweaves could resolve this blocked reprocessing. It is learning to distinguish the difference between the two cases, usually by using self knowledge and self reflection, in the moment, that will assist the clinician's decision making process.

Clinician decision making cannot be linear or simplistic. Perhaps the clinician's resonance circuit was implicitly attuning to an implicit memory network in the client identifying with the aggressor, which was accepting punishment in order to continue being protected (loved) by his father (clinician). Client and clinician would be empathically resonating with shared complimentary [66] memory networks activated through their resonance circuits.

If the clinician follows the present instructions and becomes aware of his sadistic memory network only, he might experience guilt because another dysfunctional memory network became activated chastizing him. Then the clinician interprets his memory activation as pathology, in AIP terms (unprocessed memories ="counter transference"); and miss an opportunity to continue being "optimally interactive," by noticing his sadistic memory activations were induced by the client's dissociated memory networks of need to be loved and protected by being punished by the "aggressor" first, the parent, and in the moment by the clinician by continuing to use bilateral stimulation. By the clinician first noticing his somatic activations, compartmentalizing them, identifying and decoding them and then using this inter subjective knowledge in a relational interweave [48]. Without the clinician being instructed to use his somatic activations productively, he might falsely blame himself for his sadism; seek out treatment for his counter transference and a moment of meeting would be lost due to these instructional limitations.

\section{Synergy Between EMDR Protocols and Procedures and Memory Networks}

There is also a synergy between application of EMDR procedures and memory networks of the clinician, and their receipt, compliance, confusion or opposition in the memory networks of the client. How can one apply procedure "objectively"? It can only be done within one's subjective experience of living in the moment. Procedure and memory network are inseparable.

Here are two examples. In the first case an EMDR training participant, who is excited and overconfident about his abilities believes that he has mastered the procedural issues, so much so that even after his 10 hours of consultation (though he "acted compliant" to receive his certificate of completion) believes he can make "modifications" (such as leaving out Safe/Calm place and the DES, during the preparation phase, and the PC and VoC 
during the procedural steps outline); and implicit dysfunctional memory networks, activated around issues of rebelling against authority, "in sneaky ways" feels confident that when applying EMDR procedures with "this" (complex) traumatized client there will be a good outcome. Another EMDR training participant, from the same training, is a good clinician, but dysfunctional memory networks holding painful experiences and beliefs of inadequacy believes that he hasn't sufficiently mastered EMDR procedures, even after the same 10 hours of consultation. His consultant encourages him to begin with a client he has worked with, who has adult onset PTSD; he has adaptive memory networks holding beliefs that hard work leads to mastery, and strives to master the protocols and procedures, though his dysfunctional memory networks holding beliefs about inadequacy are still quite active. Both clinicians are experienced by their respective clients' mirror neuron systems, and their clients react through their adaptive or dysfunctional memory networks.

The clinician who has "mastered" EMDR with his "superior modifications" starts EMDR Reprocessing with a client with complex PTSD, without sufficient basic preparation strategies. He begins "The EMDR" meaning phases 3-8. His client becomes pathologically dissociated, stares off into space, and by the end of the session says that he does not want to do this "EMDR" anymore. This activates this clinician's dysfunctional memory networks, and he defends against his unprocessed memory networks of defectiveness, causing him to become avoidant of trying to use EMDR methodology ever again.

In the second case, the clinician with activated inadequacy memory networks, and who believes that he has not mastered EMDR transmits these messages to his client. His client is appreciative of his willingness to try a new treatment method for trauma resolution is enthusiastic and is eager to be a coparticipant because he was a victim of $9 / 11$. In this second case the clinician (using his adaptive memory networks) opens up his manual and uses it as an aid to beginning EMDR psychotherapy with his client. They go through all the Preparation strategies; the Assessment strategies, and have an excellent session, with the client making more progress than he has before on moving to resolve this trauma. Through a mastery experience the second clinician now becomes more confident; trains in advanced EMDR methods and goes on to become an Approved Consultant. Are these situations improbable? These examples come directly from fourteen years of being a faculty member of the EMDR Institute.

This "tale of two EMDR clinicians" only captures a part of the complexity of EMDR psychotherapy. There will be multiple signals going back and forth in a nonlinear fashion between client and clinician. Becoming a competent EMDR clinician is not just about mastering AIP and the protocols and procedures of EMDR, it is about a co-creative way of maintaining mental state resonance, and noticing now moments (misattunements) as inherent parts of being human, negotiating these moments of meeting (to repair a rupture of attunement) and to restore positive resonance as a part adaptive information processing.

\section{Discussion}

Mental state resonance, [2] is part of the "tracks" the EMDR engine runs on so that the client's dysfunctional memory networks could be reprocessed and assimilated into narrative memory. This occurs when a) the clinician correctly applies EMDR procedures, and $b$ ) the adaptive memory networks of the clinician in concert with EMDR procedures. "Human connections within relationships can shape the neural connections of the brain from which mind emerges. In this manner, relationships may not only be encoded in memory, but may also shape the very circuits that enable memory to be processed and self regulation to be achieved. When secure attachment patterns begin in childhood, or when the client develops useful strategies for modulating levels of arousal during life, chances for adaptive reactions and resolutions to the stressors of living are enhanced.

In an elaborated adaptive information processing model, the clinician's adaptive and dysfunctional memory networks are intrinsically part of the EMDR approach to psychotherapy. When information processing becomes blocked the clinician can now also consider examining and reflecting on his subjectivity, and on the inter subjective matrices of dysfunctional activations of his and his client's memory networks. The Clinician Self Awareness Questionnaire $[48,67]$ is a tool clinicians find useful to those ends. However, nothing takes the place of competent EMDR psychotherapy for the clinician when he sees dysfunctional memory network activations repeating, and implicitly interrupting the work.

There are inter subjective strategies developed by this author, and alluded to, which are outside the architecture of this paper, that can be applied to use these activations productively The process of self reflection is only the first step; but it is the beginning of a series of processes that have implications for furthering the practice of EMDR psychotherapy, and using these "dysfunctional memory network activations" to productively to continue adaptive information processing, without having them be "interruptions" which necessarily require EMDR psychotherapy for the clinician. These strategies, along with this elaborated conceptualization of AIP have implications for the training of EMDR clinicians.

Selfreflection implies that the clinician is capable of maintaining dual awareness. This process may be validating for the EMDR clinician. When the clinician finds moments when dual awareness diminishes he should immediately use a compartmentalization strategy to regain his self reflective abilities. If it were not for the adaptive memory networks of the clinician, in concert with the application of EMDR procedures many dysfunctional memory networks of the client would not be reprocessed. When the clinician observes patterns of dysfunctional memory network activation which block reprocessing (in consultation with a colleague) he would be well advised to reprocess these memory networks that block mental state resonance.

Standards for training new EMDR clinicians in the United States are the responsibilities of the EMDR International Association. Slight modifications of explaining AIP might include giving explicit explanations that the formation of memory networks, from infancy is dyadic. The images, attitudes, perceptions, emotions, sensations, cognitions and beliefs that comprise adaptive memory networks are the sum total of adaptive (autobiographical inter subjective) experiences, and dysfunctional (implicitly encoded inter subjective) experiences, become dysfunctional memory networks; and that each may become activated in the present 
due to the process of association, which will then be taught in the standard way in the training. Examples could include the client clinician matrix as well. The trainer could mention that every human being who walks the planet has a combination of adaptive and dysfunctional memory networks. There could be a section in the manual where the participant could be encouraged to write out one or two adaptive and dysfunctional memories networks that still affect his current day functioning. There would be no need to share this with anyone. By doing so, this concept, which seems to elude many EMDR clinicians, may become tangible and affectively encoded in the participant's brain (My thanks to George Abbott Ph.D for this idea).

During each phase of EMDR, one or two sentences could be spoken about how the memory networks of the client could affect the memory networks of the clinician, and visa versa, starting with the adaptive memory networks of both setting the stage for mental state resonance. When mental state resonance is breached the suggestion could be given that the clinician and client together search for what caused the misattunement; this could enhance the clinician's understanding that the success of EMDR depends up both a collaborative working alliance and the attention to mindfulness of both parties. Fidelity to the protocols and procedures yields the most robust results [42] and that the participant should be mindful of the effect on the psychotherapy process should he modify them.

During the practicum experience the clinician could be encouraged to think about a client they (SUDS,5) experience some dysfunctional memory network activation with, and then use this memory activation as the present day referent, developing a targeting sequence plan from that point. This would give the clinician a sense of what they may need to reflect about not only during the training, and back in their offices.

\section{Conclusion}

The EMDR International Association is urged to incorporate as a condition of completing basic EMDR training that each participant be expected to complete a number of sessions as a client, outside of the practicum. As a psychoanalyst I know the value of having a personal analysis in order not only to work on my issues (we'll leave the discussion of utility of training analyses for another time), but here we would be just having the clinician partake in the experience that they will be performing.

The client's dysfunctional memory networks may also not be available to the verbal reflective domain of communication, and the clinician needs to learn how to use his somatic reactions (which I demonstrated in the case of "Robert.") He may be aware that his adaptive memory networks were explicitly activated, but not be aware of the possibility that his implicit dysfunctional memory networks may have also been activated as well. In treatment, sometimes dysfunctional memory networks of clients may not be available verbally, and the clinician must use his non verbal memory networks, or in Racker's terms, his "non self" (1968) to find the messages in the client's non verbal presentations.

\section{Acknowledgment}

None.

\section{Conflict of Interest}

The authors declare no conflict of interest

\section{References}

1. Trevarthen C (1979) Communication and cooperation in early infancy: A description of primary intersubjectivity. In: MM Bullowa (Eds.), Before speech: The beginning of interpersonal communication. Cambridge U. Press, New York, USA, pp. 231-347.

2. Siegel DJ (1999) The developing mind. Guilford, New York, USA.

3. Schore A (1994) Affect regulation and origin of the self. Lawrence Erlbaum Associates Inc., Hillsdale NJ, New York, USA.

4. Schore A (2003) Affect dysregulation and repair of the self. Norton, New York, USA.

5. Schore A (2003) Affect dysregulation and disorders of the self. Norton, New York, USA.

6. h t t p : / / c it e seerx.ist.psu.edu/vi e wdoc/ download?doi=10.1.1.631.9487\&rep=rep1\&type=pdf

7. LeDoux J (1996) The emotional brain. Simon and Schuster, New York, USA.

8. Fadiga L, Fogassi L, Pavesi G, Rizzolatti G (1995) Motor facilitation during action observation: a magnetic stimulation study. J Neurophysiol 73(6): 2608-2611.

9. Ferrari PF, Gallese V, Rizzolatti G, Fogassi L (2003) Mirror neurons responding to the observation of ingestive and communicative mouth actions in the monkey ventral premotor cortex. Eur J Neurosci 17(8): 1703-1714.

10. Gallese V (2001) The shared manifold hypothesis: from mirror neurons to empathy. Journal of Consciousness Studies 8: 33-50.

11. Gallese V, Eagle MN, Migone P (2005) Intentional attunement: mirror neurons and the neural underpinnings of interpersonal relations: J Am Psychoanal Assoc 55(1): 131-176.

12. Gallese V (2007) Before and below theory of mind: embedded simulation and the neural correlates of social cognition. Philos Trans R Soc Lond B Biol Sci 362(1480): 659-669.

13. Gallese V (2008) Empathy, embodied simulation and the brain. J Am Psychoanal Assoc 56(3): 769-781.

14. Rizzolatti G, Sinigaglia C (2006) Mirrors in the brain. Oxford University Press, Oxford, USA.

15. Rizzolatti G, Fadiga L, Fogassi L, Gallese V (1999) Resonance behaviors and mirror neurons. Archives Italiennes de Biologie 137(2-3): 83-99.

16. Cozolino L (2006) The neuroscience of psychotherapy. NY Norton, USA.

17. Siegel DJ (2007) The mindful brain. Norton, New York, USA.

18. Iacoboni (2008) Mirroring people: the new science of how we connect with others. Farrar, Strauss, and Giroux, New York, USA.

19. Iacoboni M, Molnar-Szakacs I, Gallese V, Buccino G, Mazziotta JC, et al. (2005) Grasping the intentions of others with one's own mirror neuron system. PLoS Biol 3(3): 529-535.

20. Shin LM, Wright CI, Cannistraro PA, Wedig MM, McMullin K, et al. (2005) A Functional Magnetic Resonance Imaging Study of Amygdala and Medial Prefrontal Cortex Responses to Overtly Presented Fearful Faces in Post Traumatic Stress Disorder. Arch Gen Psychiatry 62(3): 273-281. 
21. Damasio A (2003) Looking for spinosa. Harcourt Brace, USA, pp. 1-367.

22. Bowlby J (1969) Attachment and loss Vol 1: Attachment. NY Basic Books, New York, USA, pp. 1-326.

23. Bowlby J (1973) Attachment and loss. Vol 2, Separation, NY Basic Books, New York, USA, pp. 1-355.

24. Bowlby J (1980) Attachment and loss. Vol 3, Loss. NY Basic Books, New York, USA, pp. 1-355.

25. Cassidy J, Shaver P (1999) Handbook of attachment: theory, research and clinical applications. Guilford Press, New York, USA

26. Lyons-Ruth K (2006) From infant attachment disorganization to adult dissociation: relational adaptations or traumatic experiences In Chefez, Dissociative disorders: an expanding window into the psychobiology of the mind. Psychiatric Clinics of North America 29(1): 63-86.

27. Lyons-Ruth K (2006) The interface between attachment and intersubjectivity: Perspective from the longitudinal study of disorganized attachment. Psychoanalytic Inquiry 26(4): 595-616.

28. Lyons-Ruth K (1999) Two person unconscious: Intersubjective dialogue, enactive relational representation, and the emergence of new forms of relational organization. Psychoanal Inq 19: 576-617.

29. Solomon M, Siegel D (2003) Healing Trauma: attachment, mind, body, and brain. Norton, New York, USA.

30. Wesselman D (2007) Treating attachment issues through EMDR and a family systems approach. In: Shapiro F \& Kaslow F (Eds.), Handbook of EMDR and family therapy processes. Wiley, New York, USA.

31. Courtois C, Ford J (2009) Treating complex ptsd: am evidence based guide. NY Guilford, New York, USA.

32. Edmonds T, Sloan L, McCarty D (2004) Sexual abuse survivors' perceptions of the effectiveness of EMDR and eclectic therapy: A mixed methods study. Research on Social Work Practice 14(4): 259 272

33. Bordin ES (1979) The generalizability of the psychoanalytic concept of the working alliance. Psychotherapy: Theory Research \& Practice 16: $252-260$.

34. Safran J, Muran J, Samstag L, Stevens C (2002) Repaing alliance ruptures. J Psychotherapy relationships that work, Oxford University Press, USA.

35. Lyons-Ruth K (1998) Implicit relational knowing: Its role in development and psychoanalytic treatment. Infant Mental Heath J 19(3): 282-289.

36. Shapiro F (2001) Eye movement desensitization and reprocessing Basic principles, protocols and procedures. ( $2^{\text {nd }}$ edn), Guilford, New York, USA.

37. Shapiro F (2002) EMDR as an integrative psychotherapy approach: experts of diverse orientations explore the paradigm prism. American Psychological Association, Washington D.C.

38. Shapiro F (2006) EMDR: New notes on adaptive information processing with case formulation principles, forms, scripts and worksheets Watsonville CA. EMDR Institute, USA.

39. Shapiro F, Kaslow F, Maxfield L (2007) Handbook of EMDR and family therapy processes. Wiley, New York, USA.

40. Shapiro F (2007) EMDR, Adaptive Information Processing, and Case Conceptualization. J EMDR Practice and Research 1(2): 68-87.
41. Solomon R, Shapiro F (2008) EMDR and the adaptive information processing model: Potential Mechanisms of Change. J EMDR Practice and Research 2(4): 315-325.

42. Maxfield L, Hyer L (2002) The relationship between efficacy and methodology in studies investigating EMDR treatment of PTSD. J Clinical Psychol 58(1): 23-41.

43. Heimann P (1950) On countertransference. International Journal of Psychoanalysis 31: 81-84.

44. Fromm-Reichman F (1950) Principles of intensive psychotherapy. University Press of Chicago, Chicago, USA.

45. Little M (1960) Countertransference. British Journal of Medical Psychology. 33: 29-31.

46. Wachtel P (1977) Psychoanalysis and behavior therapy. NY Basic Books, New York, USA.

47. Gelso C, Haye J (1998) The psychotherapy relationship: Theory, research and practice. Wiley, New York, USA, pp. 304.

48. Dworkin M (2005) EMDR and the relational imperative; the therapeutic relationship in emdr treatment. Routledge, New York, USA

49. Dworkin M (2003) Integrative approaches to EMDR: empathy, the intersubjective, and the cognitive interweave. J Psychotherapy Integration 13(2): 171-187.

50. Leeds A, Shapiro F (2000) EMDR and resource installation: principles, and procedures for enhancing current functioning and resolving traumatic experiences. In: J Carlson (Ed.), Brief therapy strategies with individuals and couples. Zeig/Tucker, Phoenix, USA.

51. Korn DL, Leeds AM (2002) Preliminary evidence of efficacy for EMDR resource development and installation in the stabilization phase of treatment of complex posttraumatic stress disorder. J Clinical Psychol 58(12): 1465-1487.

52. Kluft R, Fine C (1993) Clinical perspectives on multiple personality disorders. American Psychiatric Press, Washington DC.

53. van der Kolk B, McFarleane A, Weisaeth L (Eds) (1996) Traumatic Stress. NY Guilford, New York, USA

54. Lyons-Ruth K (2000) I sense that you sense that I sense: Sander's recognition process and specificity of relational moves in the psychotherapeutic setting. Infant Mental Health Journal 21(1): 8599.

55. Ainsworth M, Blehar M, Waters E, Wall S (1978) Patterns of attachment: A psychological study of the strange situation. Erlbaum, Hillsdale NJ, USA, pp. 410.

56. Main M (1995) Recent studies in attachment: Overview, with selected implications for clinical work. In: Goldberg S \& Moire R (Eds.), In Attachment Theory: Social Developmental and Clinical Perspectives. The Analytic Press, New York, USA.

57. Barach P (1991) Multiple personality disorder as an attachment disorder. Dissociation 4: 117-123.

58. Liotti G (1992) Disorganized/disoriented attachmentin the eitiology of dissociative disorders. Dissociation 5(4): 196-204.

59. van der Hart O, Steele K, Nijenhaus E (2006) The Haunted Self. NY Norton, New York, USA.

60. Morgan A (1998) Moving along to things left undone. Infant Mental Health Journal 19(3): 324-332.

61. Bromberg P (2006) Awakening the dreamer: Clinical journeys, Analytic Press, New York, USA. 
62. Bromberg $P$ (1998) Standing in the spaces: Essays on clinical processes, trauma, and dissociation. Analytic Press, New York, USA.

63. Kiesling R, Thomas R, Laliotis D, Errebo N, Morrow R, et al. (2009) The EMDR approach to psychotherapy; Basic training course, USA.

64. Uddin LQ Iacoboni M, Lange C, Keenan JP (2007) The self and social cognition: the role of cortical midline structures and mirror neurons. Trends Cogn Sci 11(4): 153-157.
65. Locke, John (1963) The Works of John Locke, A New Edition. Thomas Tegg, London.

66. Racker H (1968) Transference and countertransference. International Universities Press, New York, USA.

67. Luber M (2009) Eye movement desensitization and reprocessing scripted protocols: Basic and special situations. Springer, New York, USA. 